\title{
A Delay Prediction Approach for Teleoperation over the Internet
}

\author{
Tissaphern Mirfakhrai and Shahram Payandeh
}

\begin{abstract}
Based on the notion of wave variables, and the idea of wave-integral transmission, a new method is suggested to match the system parameters with changes in the delay. An autoregressive model is used as a predictor to forecast the future values of the delay. The predictions are used with a look-up table to tune the gain with which the wave integrals are to be fed to the system. This gain scheduling and tuning improves the system performance and decreases the mismatch between forces and velocities at the master and slave sides.
\end{abstract}

Keywords - Teleoperation, Force-feedback, Internet.

\section{INTRODUCTION}

$\mathrm{T}$ HE concept of teleoperation been around for a while [3]. Design and development of telerobotic systems were further motivated with introduction of a new ubiquitous means of communication, i.e. the Internet. Along with general problems caused by time delays, using the Internet as the means of communication can result in more difficulties in teleoperation because of the variable time delay. The variable time delays can result in wrong command order, decreased control latency and even lost command packets.

It has been shown by Anderson and Spong [2] that if the force and velocity signals are transmitted as they are from the master side to the slave side in the presence of force feedback, the system will become unstable even with the smallest delays. They suggested a modified control law, which was based on the transfer function of a pasive transmission line, and guaranteed the system stability.

Based on Anderson's ideas, Niemeyer and others [5] proposed the concept of Wave variables. Using the wave variables will result in a two-port communication line that is passive from outside. Therefore the stability of the system during teleoperation is guaranteed.

The wave variables are introduced by redefining the system power flow. Let $\mathbf{F}$ be the force applied to a system and $\dot{\mathbf{x}}$ be the velocity of motion in that part of the system. Usually, the power flow is defined as the product of an effort and flow pair such as:

Tissaphern Mirfakhrai and Shahram Payandeh, Experimental Robotics Laboratory, Simon Fraser University, Burnaby BC, Canada V5A 1S6, Email: tmirfakh@cs.sfu.ca, shahram@cs.sfu.ca.

$$
P=\dot{\mathbf{x}}^{T} \mathbf{F}
$$

To introduce the wave variables $\mathbf{u}$ and $\mathbf{v}$, we assume two streams of power moving in opposite directions in the system. This means we have divided the power flow to a stream going from the master side towards the salve side (positive direction) $\left(\frac{1}{2} \mathbf{u}^{T} \mathbf{u}\right)$ and a stream going from slave to master $\left(\frac{1}{2} \mathbf{v}^{T} \mathbf{v}\right)$. In other words, we assume that the master side is always giving energy to the system. This given energy might become negative at instants, meaning that the power transfer is actually from slave to master.

Therefore, to introduce the wave variables, we can redefine the power flow as:

$$
P=\frac{1}{2} \mathbf{u}^{T} \mathbf{u}-\frac{1}{2} \mathbf{v}^{T} \mathbf{v}
$$

We assume that $\mathbf{u}$ and $\mathbf{v}$ are linear combinations of $\dot{\mathbf{x}}$ and $\mathbf{F}$. Using equation 1 and 2 , the wave variables can be calculated in terms of $\dot{\mathbf{x}}$ and $\mathbf{F}$ as:

$$
\mathbf{u}(t)=\frac{b \dot{\mathbf{x}}(t)+\mathbf{F}(t)}{\sqrt{2 b}} \quad \mathbf{v}(t)=\frac{b \dot{\mathbf{x}}(t)-\mathbf{F}(t)}{\sqrt{2 b}}
$$

The tuning parameter $b$ acts as a weight function and changes the relative magnitude of $\dot{\mathbf{x}}$ and $\mathbf{F}$ with respect to each other. Any pair of the above variables $(\mathbf{u}, \mathbf{v}, \dot{\mathbf{x}}, \mathbf{F})$, can be selected as input or output variables to the wave-transformer block. It is possible to show that the communication line is passive if the energy stored in the outgoing wave of $\mathbf{v}$ is limited to the energy of incoming wave of $\mathbf{u}$. Now, if these wave variables are transmitted instead of the actual force or velocity signals, the overall system would be passive and no instability will happen.

Figure 1 shows the overall block diagram of the wave-based teleoperation system. The operator applies a force $\mathbf{F}_{h}$ to the master manipulator with the mass of $M_{m}$. The master controller is a PD controller with the damping factor of $B_{m}$ and spring factor of $K_{m}$. A feedback force is generated by the master controller based on the feedback wave variables. This input force along with the feedback force, will determine the velocity of motion of the master manipulator. The master force $\left(\mathbf{F}_{m}\right)$ and master velocity $\left(\dot{\mathbf{x}}_{m}\right)$ are then coded into the wave variable $\mathbf{u}_{m}$ using equation 3 . This 
wave variable is then transmitted through the communication line to the slave side.

On the slave side the wave variable is received after going through the time delay. Then the wave variable is decoded again using equation 3 . The desired slave velocity $\left(\dot{\mathbf{x}}_{s d}\right)$ is given to the slave controller, which generates the required force on the slave manipulator. This controller is a PD controller with the damping factor of $B_{s}$ and the spring constant of $K_{s}$. The slave manipulator has a mass of $M_{s}$, and is receiving the force $\mathbf{F}_{e}$ through contact with the environment.

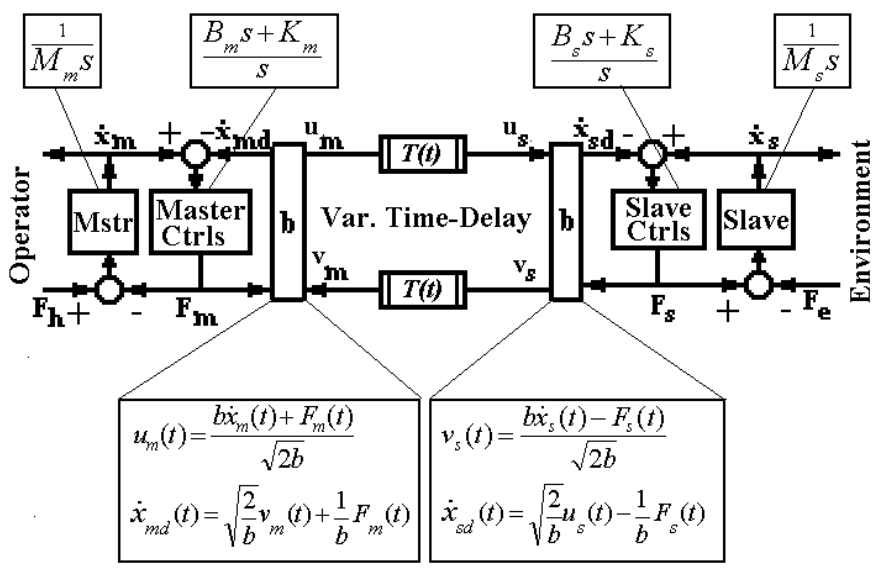

Fig. 1. The overall system block diagram.

In this paper we suggest using a delay predictor to predict the future value of delay. Introducing a delay predictor in a wave-based teleoperation system can improve the performance through feedbacking the integrals of the wave variables. By tuning the value of the feedback gain for the integrals, some of the lost properties of the signal can be restored the overall delay can be reduced for force-precision or velocity-precision tasks.

The paper is organized as follows: Section II explains the idea of transmitting the wave variable integrals along with the original wave variables to improve system performance. The integrals somehow represent the energy of the wave variables. So if the energy difference is fed back to the system withy a certain gain, the performance can be improved. Tuning this gain according to the magnitude of the time delay helps the performance, but requires a forecast knowledge of the delay value. Section III discusses our suggested method to predict the future values of the delay. In section IV, we use these predictions to tune the system through teleoperation. Section V includes some concluding remarks.

\section{Wave Integral Transmission}

Depending on the task in hand, and whether we want to match forces or velocities, there will always be some mismatch between forces and velocities at the master and slave sides. Even if the mismatch between the velocities is small, the error will cause the positions of the two manipulators to drift apart gradually as time passes.

One performance improvement strategy is transmitting wave integrals. As the wave variables themselves carry information about the forces and velocities of the two manipulators, their integrals will encode momentum and position. Thus transmitting the wave integrals means some information about the position of the manipulators. Although theoretically the position information can be decoded from the original wave variables by integrating the velocities, the position error will increase gradually because of numerical integration methods used. Transmitting the wave integrals will solve this problem.

Niemeyer and Slotine [6], had suggested to transmit the wave integrals $U(t)=\int \mathbf{u} d t$ and $E(t)=\int \mathbf{u}^{2} d t$ along with the wave variables themselves to improve the teleoperation performance. $E(t)$ is the wave energy, meaning the energy that is being transmitted by the wave stream in a certain direction.

They suggested using a filter to obtain a corrected version of $\mathbf{u}_{s}$, denoted as $\mathbf{u}_{\text {out }}$, from the integral of the wave variable $U(t)=\int \mathbf{u} d t$ and the integral of the square of the wave variable $E(t)=\int \mathbf{u}^{2} d t$ (Figure 2).

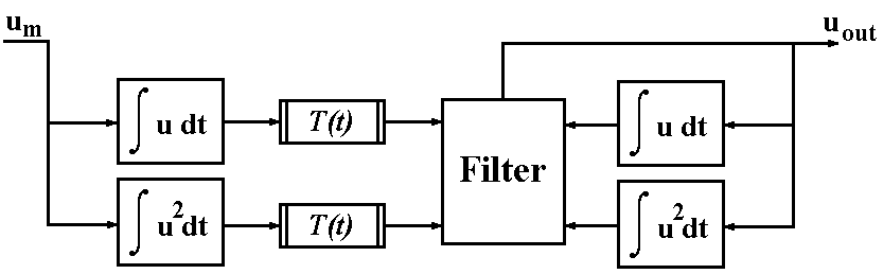

Fig. 2. The configuration of the communication link using a reconstruction filter as suggested by Niemeyer and Slotine

The filter is defined as

$$
\mathbf{u}_{\text {out }}(t)=\left\{\begin{array}{ccc}
\alpha_{\overline{U(t)}} & \text { if } & U(t) \neq 0 \\
0 & \text { if } & U(t)=0
\end{array}\right.
$$

where $\alpha$ is a constant that can modify the shape of the filter response. The filter impulse responses for $\alpha=1, \alpha=1.5$ and $\alpha=2$ are plotted in figure 3 .

The idea of transmitting wave integrals was further investigated by Yokokohji, Imaida, and Yoshikawa in [7]. They suggested that the integral of the master side wave variable $\left(\mathbf{u}_{m}\right)$ should be calculated numerically up to the time of each transmission and then should be sent along to the slave side.

At the slave side, the integral of the received wave variable $\hat{\mathbf{u}}_{s}(t)$ is calculated similarly and is then compared with the numeric value of the integral received 


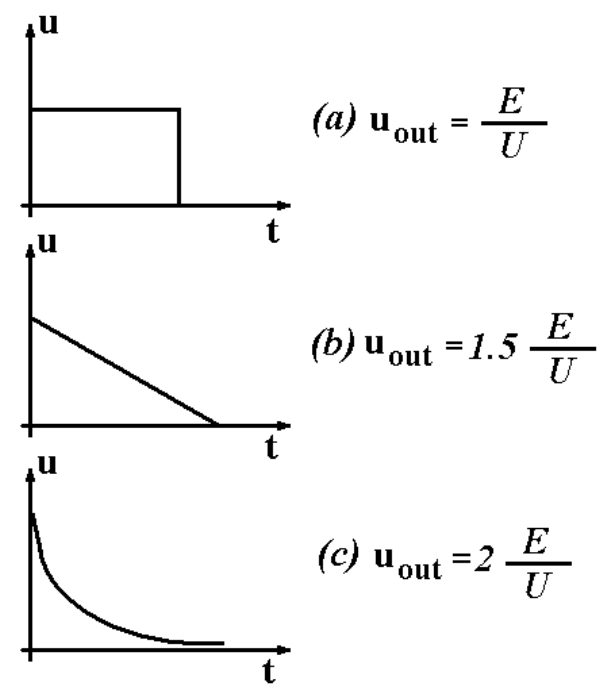

Fig. 3. The impulse response of the reconstruction filter suggested by Niemeyer and Slotine for $\alpha=1, \alpha=1.5$ and $\alpha=2$

from the master side. The difference $(\Delta)$, can be calculated as:

$$
\Delta(t)=\int \mathbf{u}_{m}(t)-\int \hat{\mathbf{u}}_{s}(t)
$$

$\Delta$ can be interpreted as a measure of change in energy of the signal, and will be fed back to $\hat{\mathbf{u}}_{s}$ to restore the lost energy (Figure 4). $\Delta(t)$ is added to the received wave variable, $\hat{\mathbf{u}}_{s}(t)$, amplified by a certain gain, hereafter called $\sigma$. Finally we calculate the slave wave variable as:

$\mathbf{u}_{s}(t)=\hat{\mathbf{u}}_{s}(t)-\sigma \Delta(t)=\mathbf{u}_{s}(t)-\sigma\left(\int \mathbf{u}_{m}(t)-\int \hat{\mathbf{u}}_{s}(t)\right)$

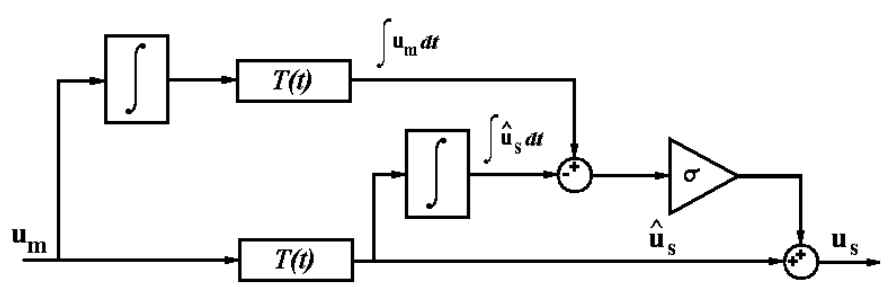

Fig. 4. The configuration of the communication link using a reconstruction filter as suggested by Niemeyer and Slotine

Yokokohji et al. [7]had noticed the importance of the gain of this feedback $(\sigma)$ and had mentioned that the value of the gain should be chosen such that the system is well compensated, but at the same time not to sensitive to disturbances. However, they had not suggested a practical way to tune this gain.
Our simulations show that the optimal value of this ${ }^{3}$ gain, to obtain the smallest error varies as the magnitude of the time delay changes. This means that at every value of $T(t)$, the error in force/velocity can be minimized if the magnitude of $\sigma$ is chosen accordingly. Therefore if the future time delay is known, a lookup table can be formed for the purpose of finding a $\sigma$ value that results in the smallest mismatch between the forces or velocities at the master and slave sides. This idea will be discussed in detail in section IV

The question now is how to obtain an a priori knowledge of the random time delay of the Internet. In other words we need to predict the future value of the delay to be able to tune the gain to its optimal value. The next section addresses the method of prediction.

\section{Modeling ANd Prediction}

The Internet time-delays are random processes. A random process is a random variable the value of which also depends on time. Although random processes are random and therefore unpredictable, models can be created based on their past values to predict the future values of the process with some error. The most widely used models for random processes are the Moving Average (MA) Model and the Autoregressive (AR) model. The moving average model acts basically as a lowpass filter on the incoming signal, and predicts the future value of the signal to be the average of its past values. However, averaging disregards all of the highly stochastic behavior of the process. Thus an MA model is not adequate for a highly stochastic process such as the Internet time delay. Therefore, the autoregressive model has to be used for the delay prediction application. For a study of alternative delay prediction approaches see [10].

\section{A. Model derivation}

An autoregressive (AR) model is a model that relates the value of the signal at time $n$ to its values at time $n-1$ through

$$
x[n]=\sum_{i=1}^{N} a_{i} x[n-i]+w[n]
$$

where $x[n]$ is the signal we want to model and $N$ is the order of our AR model, which can be selected as a design parameter[4]. $w[n]$ is white noise with autocorrelation

$$
E\left[w[n] w^{*}[n]\right]=\sigma_{w}^{2} \delta[n]
$$

where $*$ indicates complex conjugate, $\sigma_{w}^{2}$ is the variance of the noise and the operator $E[]$ takes the expected value of the parameter appearing between its brackets. 
It is desired to find the coefficients $a_{1}, a_{2}, a_{3}, \ldots a_{N}$ such that if the last $N$ values of $x$ are known $(x[n-$ $1], x[n-2], \ldots x[n-N])$, the next value of $x(x[n])$ can be predicted using equation 6 with the smallest error comparing to its actual measured value. It can be shown [4] that the optimal values of $a_{i}$ s to give the smallest error between the predicted value and the actual measured value of $x$ are the solutions of the Yule-Walker matrix equation [1].

We measured the delay from the Simon Fraser University (SFU), BC, Canada to a number of different destinations around the world. [1]. The results illustrated in the present paper were measured from the communication link to the Data Communication Incorporation (DCI) in Iran, which lies almost on the exact opposite side of the globe to SFU. As the delays of the Internet are known to have a quasi-periodic profile over a week [9], we create our model based on the delay values observed during a week. This model is then used to predict the behavior of the delay over the week after the observations. Delays are measured several times every day and a piecewise model is constructed from all sets of results.

The sampling times were arbitrarily selected to be 12:00AM, 4:00AM, 8:00AM, 12:00PM, 4:00PM and 8:00PM everyday. These particular times were selected such that they fall in different times of the day, representing very low, very high and average data traffic load. Starting at each of the above times, the delay was measured 24 times using the ping utility. The experiments were continued for 2 weeks.

For each experiment, the Root Mean Square (RMS) error was calculated for different model orders, to pick an order that gives us the smallest error. The errors versus the model order $N$ are plotted in figures 5.a and 5.b. The error seems to be decreasing when the model order $N$ is increased. To avoid unnecessarily large computation, $N=24$ was accepted as being large enough for our purpose.

Figure 6 shows 6 sets of our 24 model parameters, calculated at the six measurement times during a certain day.

\section{B. Predicting delays}

Once the model is created based on the data from measurements, the values obtained from this modeling process are used to predict the behavior of the system during the second week. This process of measuring, modeling and predicting will continue during teleoperation. In figure 7 , the predictions are compared with the actual values of the delay obtained through measurement. The prediction and the real measured values are less then \%20 different, except at times of fatal crashes at one of the master and slave computers.

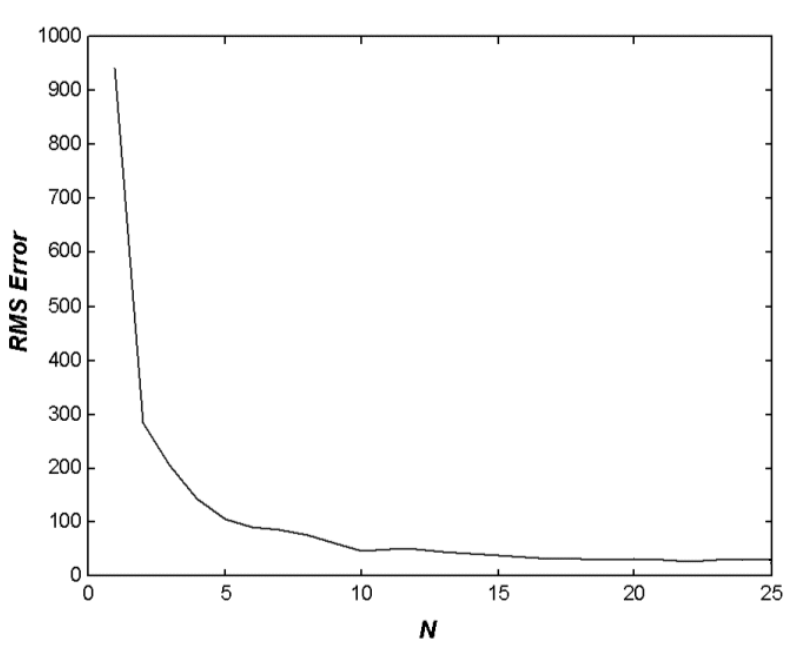

Fig. 5. Model parameters for connection with the DCI system on Saturday, plotted at the 6 measurement times.

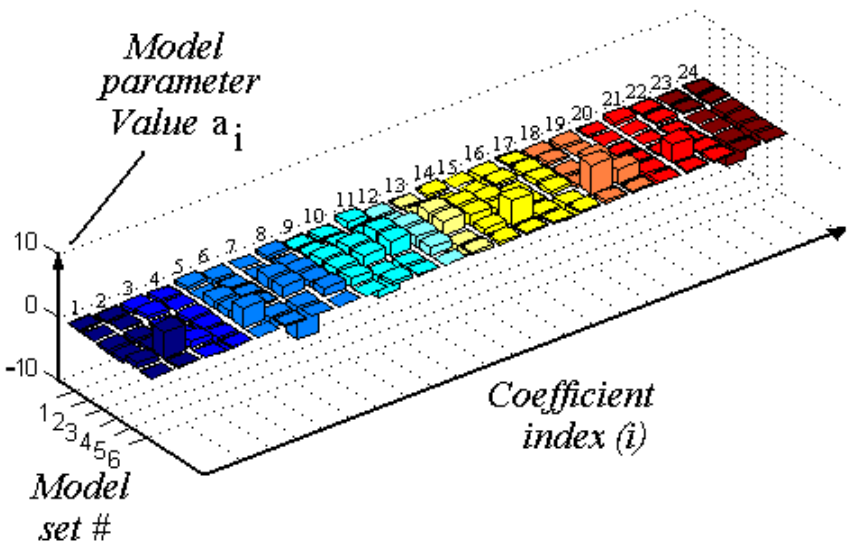

Fig. 6. Model parameters for connection with the DCI system on Saturday, plotted at the 6 measurement times.

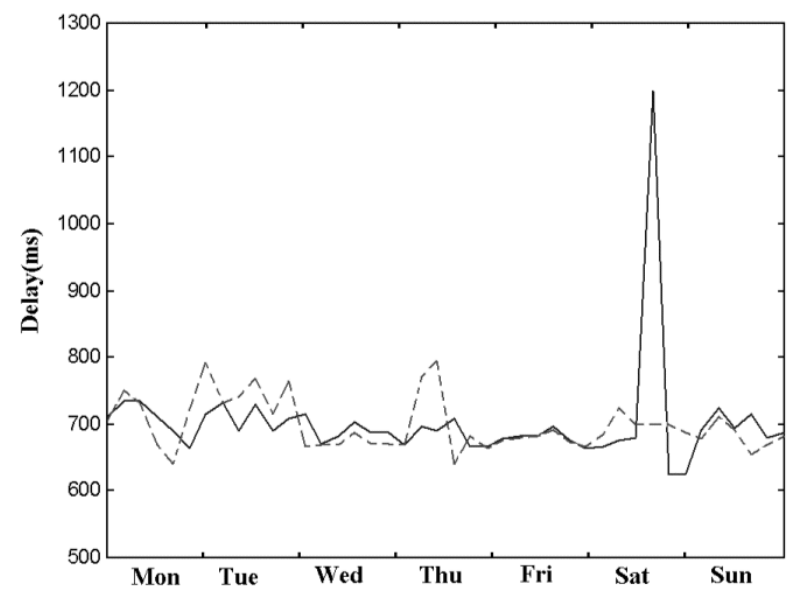

Fig. 7. Predicted delays(dashed) compared to the actual values of delays(solid) of the DCI sever during the second week.

\section{Wave Integral Gain Scheduling}

In our scenario, the operator applies a force in the form of a square pulse with the magnitude of $2 N$ to 
a master manipulator with unit mass $\left(M_{m}=1 \mathrm{Kg}\right)$. This manipulator is controlled by a PD controller with stiffness factor of $0.5 \mathrm{~N} / \mathrm{m}(\mathrm{P})$ and damping factor of $1 \mathrm{~N} / \mathrm{m} / \mathrm{s}$ (D). This controller moves the master manipulator by responding to the velocity dictated by the operator and the velocity that is obtained by the feedback system through the network.

The master side wave transformer converts the force and velocity signals to $\mathbf{u}_{m}$, which is to be transmitted through the communication line. The wave impedance $b$ is chosen to be equal to 1 , to give equal weight to the force and velocities at both master and slave sides. The delay of the communication line $(T(t))$ is assumed to be variable and will be discussed further below.

The slave side system is assumed to be exactly similar to the master side. So the slave side wave transformer works with a wave impedance also equal to 2 ; the slave manipulator also has a unit mass $\left(M_{s}=1 \mathrm{Kg}\right)$ and the controller gains are exactly the same as those of the master side. The slave manipulator is assumed to be interacting with an environment, consisting of a spring $(\mathrm{k}=1 \mathrm{~N} / \mathrm{m})$ and a damper $(\mathrm{B}=0.5 \mathrm{~N} / \mathrm{m} / \mathrm{s})$. The manipulator is pushing the spring against a solid wall, while being held by a damper.

The delay of the slave $\leftarrow$ master communication link is supposed to be equal to that of the master $\rightarrow$ slave link, i.e. $T(t)$. We also assume that there is no scaling between the master and slave sides.

Let us define the force error to be the maximum mismatch between the forces at the master side and the slave side.

$$
\mathbf{F}_{\text {err }}=\max \left\{\mathbf{F}_{m}(t)-\mathbf{F}_{s}(t-T(t))\right\}
$$

The velocity error is similarly defined as the maximum mismatch between the velocities at the master side and the slave side.

$$
\dot{\mathbf{x}}_{e r r}=\max \left\{\dot{\mathbf{x}}_{m}(t)-\dot{\mathbf{x}}_{s}(t-T(t))\right\}
$$

Our simulation studies show that for every value of $T$, there is a value of $\sigma$ to minimize the error.

Figure 8 shows the behavior of the above mentioned errors with changes in $T$ and $\sigma$. It can be seen that for every value of $T$, the value of $\sigma$ can be chosen such that the error is minimized. For each $T$ in figure 8, the top surface of one of the error bars is painted black to show the optimal value of $\sigma$. In the same figure, the hatched squares show some limitations, where the system becomes unstable due to the choice of an improper $\sigma$. At those values of $\sigma$ the amount of energy fed to the system for compensation is more than necessary and that makes the system non-passive and unstable.

When operating, the delay predictor will estimate the future value of the delay. This estimated value of
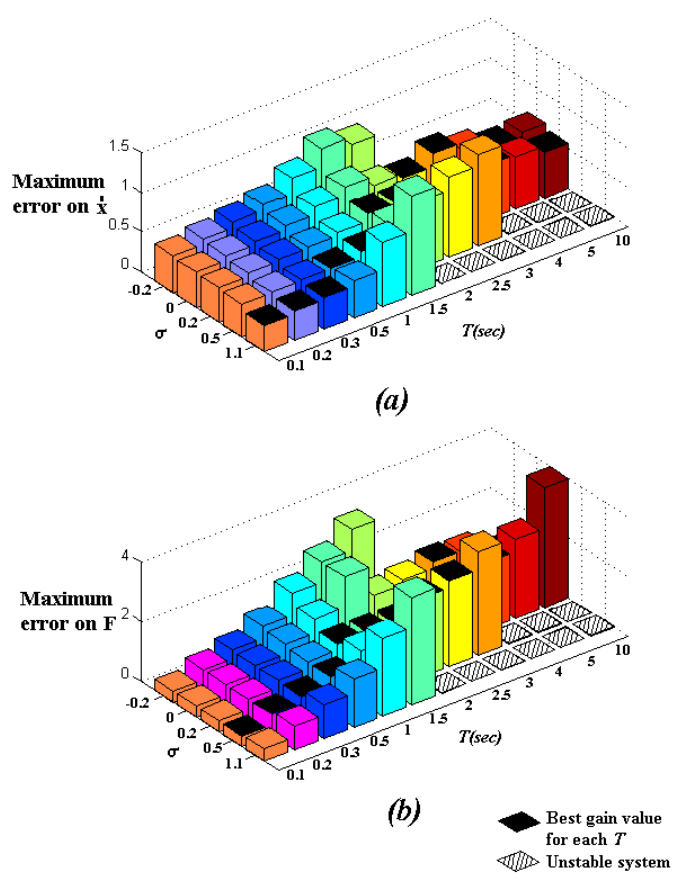

Fig. 8. Force and velocity errors at different estimated delays for different values of $\sigma$.

$T$ is then used by the gain scheduler to search in a look-up table like figure 8 to find the optimal value of the gain $\sigma$. Figure 9, compares the system performance with the optimal value of $\sigma$ with the system performance with no compensation. It is visible that the salve force follows the desired force more closely when compensation is added.

It should be noted that the value of $\sigma$ to minimize the force error, is not always the same as the value of $\sigma$ to minimize the velocity error. This can be seen from figure 8 , where the black painted path on the force error plot(8.b), is different from the black painted path on the velocity error plot(8.a).

Figure 10 shows the velocities on the master and slave sides, with and without a compensation gain that minimizes the force error. It can be seen that although applying the compensation gain of $\sigma=0.5$ has the best compensation effect on the force, it actually increases the difference between $\dot{x}_{m}$ and $\dot{x}_{s}$.

Also at large delays, if the $\sigma$ value exceeds a certain value the system goes unstable. The hatched squares in figure 8 represent such cases. This instability can be explained by noting that adding the $\sigma$-path, we are violating the conditions of passivity as stated in Niemeyer's calculations in [8]. Thus if we add too much feedback to the system, we might end up with positive feedback, which tends to destabilize the system. 

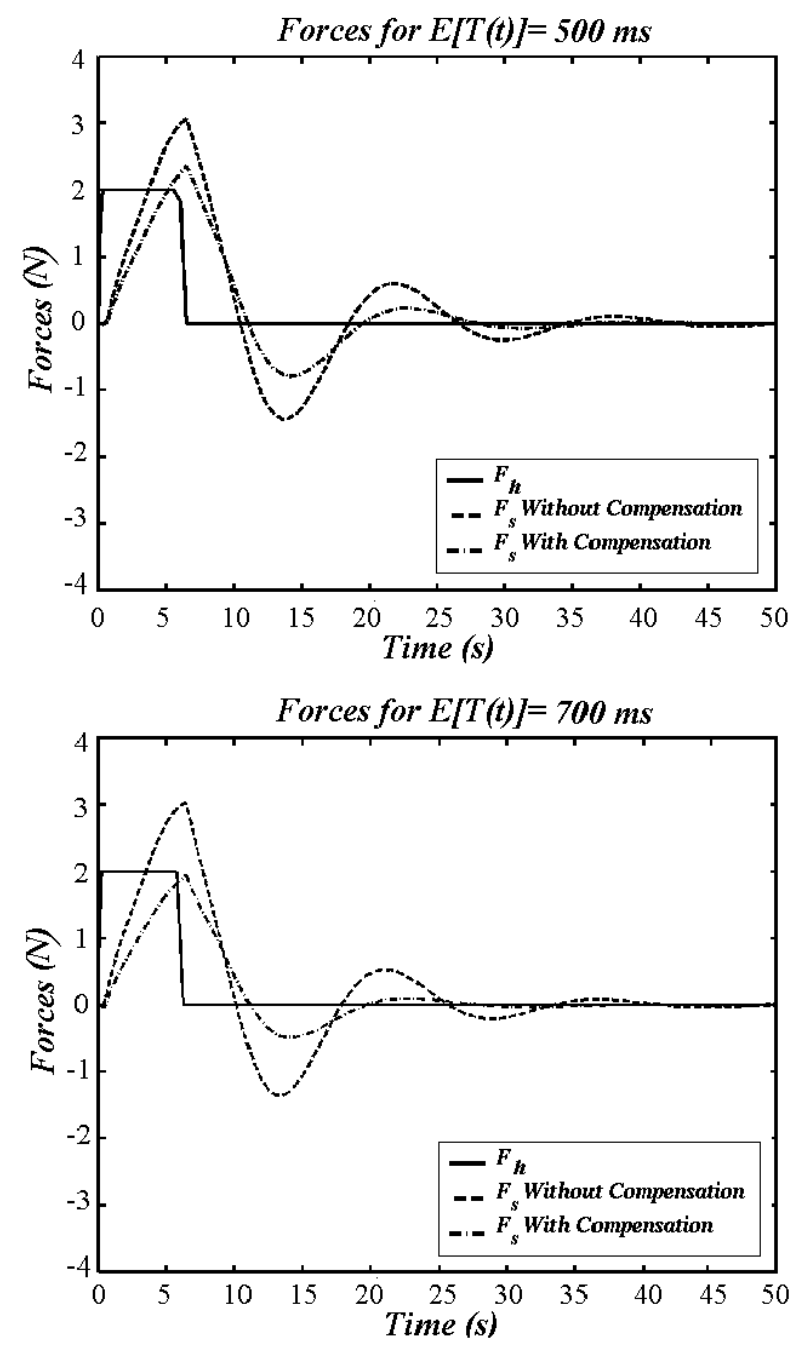

Fig. 9. Forces at $E(T(t))=500 \mathrm{~ms}$ and $E(T(t))=700 \mathrm{~ms}$

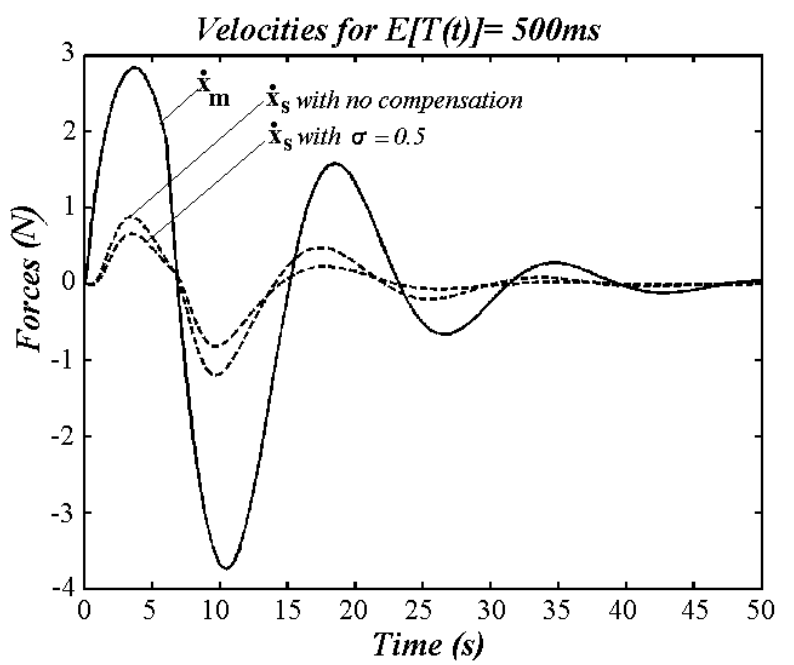

Fig. 10. $\dot{x}_{m}, \dot{x}_{s}$ without compensation and $\dot{x}_{s}$ with $\sigma=0.5$ that minimizes the force error.
Therefore, when the future value of the delay is predicted, we can use a look-up table similar to the black path in figure 8 to retune the system. This way choosing the optimal value of $\sigma$ can minimize our error on force or velocity, or both. When dealing with large delays the value of $\sigma$ has to be set to zero, to guaranty the passivity of the system and to keep stable operation. For example, if the predicted value for $T(t)$ is 1 second, it is optimal to set $\sigma=0.5$ based on figure 8.a if a minimal force error is intended. Also $\sigma=0.2$ will result in minimal velocity error as is visible from figure 8.b.

\section{Conclusions}

A method is suggested to decrease the error between forces/velocities of the master and slave manipulator. The future value of the variable time delay is predicted based on its past values using an Autoregressive (AR) model. A look-up table is then used to find a value of the wave integral feed-forward gain $\sigma$ to minimize the error between the force/velocities of the master and slave manipulators. By tuning the gain to this optimal value, some of the lost properties of the signal can be restored, the error between the master and slave forces/velocities is decreased and the overall teleoperation system performance is improved.

\section{REFERENCES}

[1] Tissaphern Mirfakhrai and Shahram Payandeh, A Model for Time-Delays for Teleoperation over the Internet, Proc. IEEE Symposium of Computational Intelligence in Robotics and Automation(CIRA 2001), pp.236-241.

[2] Robert J. Anderson and Mark W. Spong, "Bilateral control of Teleoperators with time delay, IEEE transactions on Automatic Control, 34(5):494-501, 1989.

[3] Thomas B. Sheridan, Space teleoperation through time delay review and prognosis, IEEE Transactions on Robotics and Automation, 9(5):592-606, October 1993.

[4] Henry Stark, John W. Woods, Probability, Random Processes, and Estimation Theory for Engineers, January 1994, Prentice-Hall Canada, ISBN 0137287917.

[5] Günter Niemeyer and Jean-Jacques E. Slotine, Stable Adaptive Teleoperation IEEE Journal of Oceanic Engineering, Vol.16, No.1, pp. 152-162, 1991.

[6] Gunter Niemeyer and Jean-Jacques E. Slotine, Towards Force-Reflecting Teleoperation Over the Internet, Proc. IEEE Int. Conf. on Robotics and Automation, pp. 19091915, 1998.

[7] Yasuyoshi Yokokohji, Takashi Imaida, and Tsuneo Yoshikawa, Bilateral Control with Energy Balance Monitoring Under Time-Varying Communication Delay, Proc. IEEE Int. Conf. on Robotics and Automation, pp. 2684$2689,2000$.

[8] Günter Dieter Niemeyer, Using wave variables in time delayed force reflecting teleoperation, PHD thesis, MIT, MA.

[9] Roberto Oboe and Paolo Fiorini, A Design and Control Environment for Internet-Based Telerobotics, The International journal of Robotics Research, vol.17, No. 4, April 1998, pp. 433-449.

[10] Q.P.Wang, D.L.Tan, Ning Xi and Y.C.Wang, The Control Oriented QoS: Analysis and Prediction, Proc. of the 2001 IEEE International Conference on Robotics \& Automation, pp. 1897-1902. 